\title{
Time-on-task effects in children with and without ADHD: depletion of executive resources or depletion of motivation?
}

\author{
Tycho J. Dekkers ${ }^{1,2} \cdot$ Joost A. Agelink van Rentergem ${ }^{1} \cdot$ Alette Koole $^{1} \cdot$ \\ Wery P. M. van den Wildenberg ${ }^{1,3} \cdot$ Arne Popma ${ }^{2,4,5} \cdot$ Anika Bexkens $^{6,7}$. \\ Reino Stoffelsen $^{2,4} \cdot$ Anouk Diekmann ${ }^{2,8} \cdot$ Hilde M. Huizenga ${ }^{1,3,9}$
}

Received: 13 June 2016 / Accepted: 17 May 2017 / Published online: 23 May 2017

(c) The Author(s) 2017. This article is an open access publication

\begin{abstract}
Children with attention-deficit/hyperactivity disorder (ADHD) are characterized by deficits in their executive functioning and motivation. In addition, these children are characterized by a decline in performance as time-on-task increases (i.e., time-on-task effects). However, it is unknown whether these time-on-task effects should be attributed to deficits in executive functioning or to deficits in motivation. Some studies in typically developing (TD) adults indicated that time-on-task effects should be interpreted as depletion of executive resources, but other studies suggested that they represent depletion of motivation. We, therefore, investigated, in children with and without ADHD, whether there were time-on-task effects on executive functions, such as inhibition and (in)attention, and whether these were best explained by depletion of executive resources or depletion of motivation. The stop-signal task (SST), which generates both indices of inhibition
\end{abstract}

Electronic supplementary material The online version of this article (doi:10.1007/s00787-017-1006-y) contains supplementary material, which is available to authorized users.

\section{Tycho J. Dekkers}

t.j.dekkers@uva.nl

1 Department of Psychology, University of Amsterdam, Nieuwe Achtergracht 129B, 1018 WS Amsterdam, The Netherlands

2 Department of Forensic Psychiatry and Complex Behavioral Disorders, Academic Center for Child and Adolescent Psychiatry, De Bascule, Rijksstraatweg 145, 1115 AP Duivendrecht, The Netherlands

3 Amsterdam Brain and Cognition Center, University of Amsterdam, Amsterdam, The Netherlands

4 Department of Child and Adolescent Psychiatry, VU University Medical Center Amsterdam, Amsterdam, The Netherlands (stop-signal reaction time) and attention (reaction time variability and errors), was administered in 96 children (42 ADHD, 54 TD controls; aged 9-13). To differentiate between depletion of resources and depletion of motivation, the SST was administered twice. Half of the participants was reinforced during second task performance, potentially counteracting depletion of motivation. Multilevel analyses indicated that children with ADHD were more affected by time-on-task than controls on two measures of inattention, but not on inhibition. In the ADHD group, reinforcement only improved performance on one index of attention (i.e., reaction time variability). The current findings suggest that time-on-task effects in children with ADHD occur specifically in the attentional domain, and seem to originate in both depletion of executive resources and depletion of motivation. Clinical implications for diagnostics, psychoeducation, and intervention are discussed.

Keywords ADHD - Executive functioning - Depletion . Time-on-task · Reinforcement · Inhibition

5 Faculty of Law, Institute of Criminal Law and Criminology, Leiden University, Leiden, The Netherlands

6 Department of Developmental and Educational Psychology, Leiden University, Wassenaarseweg 52, 2333 AK Leiden, The Netherlands

7 Department of Child and Adolescent Psychiatry, GGZ Delfland, Center for Psychiatry, Amsterdam, The Netherlands

8 Practice for Individual, Couple, and Family Therapy and Center for Training, De Kontekst, Van Breestraat 147HS, 1071 ZL Amsterdam, The Netherlands

9 Research priority Area Yield, University of Amsterdam, Amsterdam, The Netherlands 


\section{Introduction}

Children with attention-deficit/hyperactivity disorder (ADHD) are characterized by inattention, hyperactivity, and/or impulsivity, which lead to problems in multiple domains. For example, children with ADHD have more academic problems [1] and adverse health outcomes [2], report lower quality of life [3], and usually have one or more comorbid psychiatric diagnoses [4]. Several models explaining ADHD have been proposed (see [5, 6]). One influential model is the dual pathway model, in which ADHD is characterized by deficits in both executive and motivational systems [7].

With regard to the executive pathway, several metaanalyses indicate that children with ADHD are impaired on multiple executive functions (EF) [8-11]. For example, response inhibition, which is regarded as one of the core, higher order executive functions [12, 13], has repeatedly shown to be implicated with ADHD [11, 14]. On a more basic pre-executive level, attention is a crucial prerequisite of executive functioning [13], and associations between ADHD and attentional problems are consistently reported (ranging from problems in sustaining attention on lab tasks to real life attention problems [9, 14]).

With respect to the motivational pathway, many empirical studies as well as theoretical models suggest aberrant motivation in children with ADHD (see [15] for an overview). Some models propose that children with ADHD have a higher reward sensitivity than controls (i.e., larger improvement in performance related to reward; $[16,17])$, but experimental findings for this account are mixed [18]. However, a recent meta-analysis on reinforcement effects on inhibition in ADHD indicated that (1) a large majority of children, both with and without ADHD, benefited from reinforcement and (2) this reinforcement effect was stronger for ADHD (large effect size) than for controls (medium effect size), suggesting differential reward sensitivity between groups [16]. The authors note that only $24 \%$ of the studies found significant group $\times$ reinforcement interactions in this direction, which is in line with the mixed findings that were mentioned previously.

EF performance in children with ADHD is often more characterized by a stronger decrease in performance over time (time-on-task) as compared to TD controls [19]. It has been argued that these time-on-task effects originate in difficulties sustaining attention, which is a typical, although not specific [20], feature of ADHD [14, 21]. In accordance with the dual pathway model, this time-ontask effect can be caused by degraded (EF) resources, but it may also be possible that decreased levels of motivation explain this decrease in performance. It was shown that time-on-task effects on working memory in ADHD could be partly counteracted with reinforcement [22], suggesting that they should at least partly be attributed to decreased motivation. However, to our knowledge, it has never been tested before whether this is also the case for response inhibition and attention. Therefore, the current study investigates whether time-on-task effects on inhibition and attention in children with ADHD can be remedied by increasing motivation.

Dual pathway models of ADHD do not directly speak to the role of motivation on time-on-task effects. However, the effect of motivation on time-on-task effects is central in the literature on resource depletion in healthy adults. Some resource depletion theorists argue that self-control capacities, a concept highly related to EF [23], are limited, and consequently, self-control performance degrades after successive attempts (for reviews, see [24, 25]). However, others have argued that a decline in motivational resources (i.e., "reduced motivation to attain task goals" [26]) can also explain timeon-task effects [27, 28], as these effects appear to be weaker if participants are motivated [26, 29].

To sum up, the current study combines dual pathway models of ADHD and resource depletion models of time-on-task effects in healthy adults, to assess the origin of time-on-task effects in children with ADHD. That is, we test whether children with ADHD are more affected by time-on-task effects than TD children. To investigate the nature of these time-ontask effects, depletion of resources and depletion of motivation were disentangled. Children with and without ADHD performed twice on the stop-signal task (SST; [30, 31]), which yields a measure of response inhibition and more indirect measures of (in)attention. In the second task, participants were either assigned to a reinforced or a non-reinforced condition.

First, we hypothesize degraded performance of children with ADHD as compared to TD children in the first task and in the second task without reinforcement (effects of group) [8-11]. Second, we hypothesize degraded performance on the second task without reinforcement as compared to the first task (effect of time-on-task), and we expect this effect to be larger in children with ADHD than in TD controls (time $\times$ group interaction; [19]). Third, we hypothesize a better performance on the second task with reinforcement as compared to the second task without reinforcement (effect of reinforcement), and we hypothesize children with ADHD profit more from reinforcement than TD controls (reinforcement $\times$ group interaction; [16, 22]).

\section{Method}

\section{Participants}

ADHD participants were recruited from an academic outpatient mental healthcare center and TD control 
participants were recruited from elementary schools. In the ADHD group, children were included when they were diagnosed with ADHD (all subtypes), according to the assessment by expert psychologists or psychiatrists from the academic outpatient mental healthcare center, following DSM-IV-TR criteria [32]. There was no exclusion based on other disorders. Children in the control group were included only when their primary caretakers confirmed that there was no ADHD diagnosis. In total, our sample consisted of 111 children aged between 9 and 13 years. 54 children with ADHD (45 boys, mean age 11.2 years, $\mathrm{SD}=1.04$ ) and 57 children without ADHD (27 boys, mean age 11.8 years, $\mathrm{SD}=0.68$ ) were included.

When using stimulant medication, participants were instructed not to take their medication on the day of testing, to reach total washout [33]. Informed consent was obtained from primary caretakers of all children. All procedures were in accordance with the ethical standards of the institutional research committee and with the 1964 Helsinki declaration and its later amendments.

\section{Materials}

Response inhibition and, indirectly, attention were measured with the standard stop-signal task (SST; [30, 31]), which is a reliable indicator of inhibition in children with ADHD [34]. Several studies showed associations between the SST and a wide range of real life behaviors, e.g., associations with classroom observations of children with ADHD [35], with teacher ratings of inattention [36], with observations as well as classroom measures of hyperactivity and inattention [37], and with inattention measured by both parents and teachers [38].

The SST was administered twice (i.e., T1 and T2), both administrations consisted of one practice block and four experimental blocks of 56 trials each. In this task, children have to press one out of two marked buttons on the keyboard, corresponding to green go signals appearing in the center of the screen, as fast and accurate as possible (i.e., the go task). In $\mathrm{T} 1[\mathrm{X}]$ required a left response and $[\mathrm{O}]$ required a right response; these were replaced by $[\mathrm{H}]$ and [S], respectively, at T2 to prevent learning effects. A choice between two response keys is necessary to create time to process a potential stop signal. This presentation order was counterbalanced across participants (the first, third, fifth, etc. participant was assigned to $[\mathrm{XO}]$ at $\mathrm{T} 1$ and [HS] at T2, and in the second, fourth etc. participant, this was reversed). In $25 \%$ of the cases, the green go signal turned red, indicating that the response tendency had to be inhibited. To ensure that participants succeeded to inhibit their response in $50 \%$ of the cases, the time between the go signal and the stop signal was adaptive. That is, if stopping was successful, the interval between the go signal and the stop signal (i.e., the stop-signal delay) of the following stop trial was increased by $50 \mathrm{~ms}$, making it harder to inhibit. On the other hand, if the participant failed to stop, the stop-signal delay of the following stop trial was shortened by $50 \mathrm{~ms}$, making inhibition easier. Accordingly, the stop-signal reaction time (SSRT) can be estimated. This reflects the estimated mean time required to inhibit responses to stop signals, implying that a short SSRT indicates good inhibitory capacities. SSRT was calculated according to the integration method and the race model [41]. Assuming independence between go and stop processes, the finishing time of the stop process bisects the go RT distribution. Given that the response could not be stopped successfully on nth percent of all stop trials, SSRT is calculated by subtracting the mean stop-signal delay from the go RT that represents the nth percentile of go RTs (i.e., the finishing time of the stop process).

Furthermore, the SST provides an index of choice errors (i.e., pressing the wrong button), omission errors on go trials (i.e., no response within the response frame), and reaction time (including its variance). More omission errors, slower mean RT, and higher RT variability (defined as the standard deviation of all RTs of a participant within the SST) are associated with problems in the domain of attention [39]. More specifically, omission errors on a Go/NoGo task are related to symptoms of inattention in ADHD as reported by both caregivers and teachers [40]. Moreover, ADHD is characterized by attentional lapses, which generate reaction time distributions with a positive skew, leading to increased mean reaction times and higher RT variability [41, 42]. Relatedly, attention lapses appear to be related to errors and variability of reaction times, which is referred to as state instability [43]. Therefore, omission errors, RT and RT variability were taken as indirect measures of the basic attentional processes.

\section{Procedure}

To induce time-on-task effects, the SST was administered twice in succession. Before every block, children were instructed to respond as fast and accurate as possible to go trials and to withhold their response if the signal turned red (i.e., not to press a button). To avoid adoptation of a waiting strategy, participants were also told that it was not allowed to wait for the signal to become red. In the second task, participants were randomly assigned to either a reinforcing or a non-reinforcing condition. In the reinforcing condition, children were told that they could earn coins which could be used to "buy" a present at the end of the task if they had 
earned at least ten coins. Reinforcement was not aimed at any specific aspect of the SST, and children were instructed to respond as fast and accurate as possible. Although the present was emotionally appealing for the participants, the monetary value was about 0.50 euro. To motivate participants, the box with presents was already shown before the beginning of the second task. After each block, children were informed on the screen about the amount of earned coins. Feedback on the amount of earned coins was manipulated; the cumulative amount of coins shown after each block was, respectively, 2, 5, 7, and 9 or $10 .^{1}$ There was no possibility of losing in both conditions. The duration of one administration of the SST was approximately $16 \mathrm{~min}$; the duration of the entire session ranged from 45 to $60 \mathrm{~min}$. The time between the end of the first and the beginning of the second administration of the SST was approximately 2 min.

\section{Data analysis}

A repeated measures design with one within-subjects factor (time, T1 and T2) and two between-subjects factors (ADHD vs. TD controls; reinforcement vs. no reinforcement at T2) was used. Note, however, that the design is not fully crossed, as none of the participants was reinforced during the first task. A fully crossed design would have led to power difficulties, given the limited availability of participants. Therefore, the current data could not be analyzed with a regular repeated measures analysis, but were analyzed with a multilevel analysis with time as a first level variable and group and reinforcement as second level variables [44]. Age was added as covariate, as it might be related to executive functioning [45]. Gender and intelligence were not added as covariates, because a higher proportion of boys and a lower average intelligence level are typical features of ADHD as compared to controls [46], and are, therefore, inherently not suitable as covariates [47] (see Appendix 1 for the complete multilevel model).

Five dependent variables were derived from the SST (see "Materials" section). Note that for the omission and choice errors, the square root of the raw scores was analyzed, because percentages generally are not normally distributed.

\footnotetext{
1 The end score was manipulated to either 9 or 10 . This in order to prevent the spread of the rumor that all children had won a present regardless of their performance, as TD controls usually had class together. After all data were gathered, children that ended up with 9 points received a present as well.
}

\section{Results}

\section{Exclusion of participants}

15 participants were excluded from the analyses (12 boys, 3 girls; mean age 11.2 years, $\mathrm{SD}=1.18$ ). Four of them were excluded due to procedural errors. In addition, 11 participants were excluded because of aberrant performance: one ADHD participant refused to continue with the second task; one ADHD participant made choice errors on more than $50 \%$ of the go trials (at T1), indicating difficulty in understanding the task; one ADHD participant had a very abnormal response pattern in which RT variability was larger than mean RT (at T2 without reinforcement); four participants were excluded, because SSRT was below $100 \mathrm{~ms}$, indicating that SSRT estimation was not reliable for these participants (one ADHD participant at T1; two ADHD participants at T2, without reinforcement; and one TD participant at T2 with reinforcement); four participants were excluded, because the standardized value of at least one of the outcome measures was more than three standard deviations from the average (one ADHD participant had abnormal slow reaction times at $\mathrm{T} 1$ as well as $\mathrm{T} 2$ without reinforcement ${ }^{2}$ one ADHD participant had an abnormal high SSRT at $\mathrm{T} 1 ;^{3}$ one ADHD participant had an abnormal high SSRT at T2, ${ }^{4}$ without reinforcement; and one TD participant had an abnormal high SSRT at T2, ${ }^{5}$ without reinforcement).

Interestingly, 9 of the 11 participants excluded because of aberrant performance were diagnosed with ADHD. Most of these participants were excluded because of aberrant performance in the second task without reinforcement: of the seven exclusions based on performance at $\mathrm{T} 2$, two were reinforced, and five were not.

\section{Demographics}

After exclusion, the sample consisted of 96 participants (42 ADHD, 54 TD children). Differences between the ADHD group and the TD control group were significant with regard to age. The mean age was $11.2(\mathrm{SD}=1.0)$ and 11.8 ( $\mathrm{SD}=0.68$ ) years for ADHD and TD controls, respectively: $t(94)=3.21, p=0.002)$. Therefore, age was added as covariate in all further analyses. Groups also differed in gender ( $83 \%$ vs. $46 \%$ boys in ADHD and TD, respectively: $\left.\chi^{2}(1)=13.8, p<0.001\right)$. For extra

\footnotetext{
${ }^{2}$ Mean reaction times of this participant were 1288 and $1219 \mathrm{~ms}$ at $\mathrm{T} 1$ and $\mathrm{T} 2$, respectively.

${ }^{3}$ SSRT at T1 of this participant was $678 \mathrm{~ms}$.

${ }^{4}$ SSRT at T2 of this participant was $677 \mathrm{~ms}$.

${ }^{5}$ SSRT at T2 of this participant was $538 \mathrm{~ms}$.
} 
Table 1 Group means at T1 and at T2, with and without reinforcement

\begin{tabular}{lllllll}
\hline Measure & TD (T1) & TD (T2) - & TD (T2) + & ADHD (T1) & ADHD (T2) - & ADHD (T2) + \\
\hline Number of participants & 54 & 28 & 26 & 42 & 16 & 26 \\
Reaction time in ms (SD) & $730(175)$ & $670(130)$ & $712(228)$ & $730(161)$ & $741(98)$ & $767(206)$ \\
Reaction time variability in ms (SD) & $203(52)$ & $192(44)$ & $177(49)$ & $237(48)$ & $254(45)$ & $230(58)$ \\
$\%$ choice errors (SD) & $3.49(3.91)$ & $3.38(3.46)$ & $3.27(3.18)$ & $5.90(5.34)$ & $4.76(3.76)$ & $5.24(4.64)$ \\
$\%$ omission errors (SD) & $2.38(3.13)$ & $0.98(1.34)$ & $2.40(3.74)$ & $3.83(3.74)$ & $5.84(5.73)$ & $5.91(6.17)$ \\
SSRT in ms (SD) & $254(65)$ & $260(53)$ & $246(62)$ & $282(75)$ & $269(54)$ & $243(54)$ \\
\hline
\end{tabular}

Symbols + and - represent conditions with and without reinforcement

Note that this table depicts the $\%$ of choice and omission errors, whereas the square root is used in the analyses. Note that the number of ADHD participants in the conditions without and with reinforcement is unequal, as a relatively high number of participants that did not receive feedback were excluded because of aberrant performance. Reaction time variability reflects the mean within subject variability in RTs, whereas the SD of reaction time reflects the standard deviation of the mean RTs of all subjects

$T D$ typically developing control group, $A D H D$ attention-deficit/hyperactivity disorder, $m s$ milliseconds, $S S R T$ stop-signal reaction time, $S D$ standard deviation

analyses with gender added as covariate, see Appendix $2 .^{6}$

In the ADHD group, 31 children were diagnosed with the combined type, 5 with the inattentive type, 2 with the hyperactive-impulsive type, and 4 children were diagnosed with ADHD not otherwise specified. With regard to comorbidity, 24 children had no comorbid diagnosis, 7 children had a comorbid learning disorder, 5 had a comorbid disruptive behavior disorder (i.e., conduct disorder, oppositional defiant disorder, or disruptive behavior disorder not otherwise specified), $3 \mathrm{had}$ a comorbid parent-child relational problem, 2 had a comorbid mood disorder, and 1 had a comorbid communication disorder.

\section{Effects of group: ADHD vs. TD controls (T1)}

To test our first hypothesis that EF on $\mathrm{T} 1$ is impaired in children with ADHD as compared to TD controls, we performed ANCOVAs (and thus not a multilevel analysis) for all outcome measures in which we controlled for age differences between groups. The assumption of parallel regression lines was not violated for all five outcome measures $(p>0.05)$. ANCOVAs revealed only group differences at baseline for reaction time variability $[F(1,93)=10.8$, $p=0.001]$ and omission errors $[F(1,93)=4.5, p=0.04]$. No differences were found for SSRT $[F(1,93)=2.7$, $p=0.11]$, mean reaction time $[F(1,93)=0.001$, $p=0.98]$, and choice errors $[F(1,93)=2.9, p=0.10]$ (see Table 1 for means and standard deviations at T1).

After the baseline assessment at $\mathrm{T} 1$, participants were randomly assigned to the second task with or without

\footnotetext{
${ }^{6}$ Note however that the addition of gender as covariate yielded no differences in the results of the multilevel analyses (see extra analyses in Appendix 2).
}

reinforcement. At baseline, there were no significant differences on all five outcome measures between children assigned to the reinforced and non-reinforced second task. This was tested by adding the reinforcement condition as an additional between factor to the ANCOVAs.

\section{Multilevel analyses (also see Table 2)}

\section{Effects of group: ADHD vs. TD controls (T2: not} reinforced)

When comparing ADHD and TD control groups at T2 (without reinforcement), the multilevel analysis indicated that participants with ADHD had a larger reaction time variability $[t(119.62)=-5.04, p<0.001]$ and made more omission errors $[t(124.88)=-4.70, p<0.001]$. No differences between ADHD and TD controls at T2 were found for SSRT, mean reaction time, and choice errors.

\section{Time-on-task effects in ADHD (T2 vs. T1: not reinforced)}

Children with ADHD were characterized by increased reaction time variability $[t(102.07)=-2.25, p=0.027]$ and more omission errors $[t(96.86)=-2.64, p=0.010]$ on the second task without reinforcement, as compared to the first task. No differences between T2 and T1 in ADHD were found for SSRT mean reaction time and choice errors. Time-on-task effects were thus only found on indices of attention, but not on inhibition.

Time-on-task effects in ADHD vs. TD controls (T2 vs. T1: not reinforced)

A significant time $\times$ group interaction was observed for reaction time variability $[t(102.28)=2.80, p=0.006]$. As shown above, in the ADHD group, reaction time 
Table 2 Overview of all effects in the multilevel model

\begin{tabular}{|c|c|c|c|c|c|c|}
\hline $\begin{array}{l}\gamma \\
\text { Variable }\end{array}$ & $\begin{array}{l}\gamma_{01} \\
\text { Group }\end{array}$ & $\begin{array}{l}\gamma_{10} \\
\text { Time }\end{array}$ & $\begin{array}{l}\gamma_{11} \\
\text { Group } \times \text { time }\end{array}$ & $\begin{array}{l}\gamma_{02} \\
\text { Reinforcement }\end{array}$ & $\begin{array}{l}\gamma_{03} \\
\text { Group * reinforce- } \\
\text { ment }\end{array}$ & $\begin{array}{l}\gamma_{04} \\
\text { Age }\end{array}$ \\
\hline SSRT & $\begin{array}{c}B=8.2(16.2) \\
p=0.61\end{array}$ & $\begin{array}{c}B=23.1(12.6) \\
p=0.07\end{array}$ & $\begin{array}{c}B=-30.7(16.1) \\
p=0.06\end{array}$ & $\begin{array}{c}B=-9.8(14.3) \\
p=0.50\end{array}$ & $\begin{array}{c}B=-7.4(18.9) \\
p=0.70\end{array}$ & $\begin{array}{c}B=-0.90(0.55) \\
p=0.11\end{array}$ \\
\hline Mean RT & $\begin{array}{l}B=-58.0(49.1) \\
\quad p=0.24\end{array}$ & $\begin{array}{c}B=7.8(32.5) \\
\quad p=0.81\end{array}$ & $\begin{array}{l}B=58.6(40.8) \\
\quad p=0.15\end{array}$ & $\begin{array}{l}B=56.8(40.4) \\
p=0.16\end{array}$ & $\begin{array}{l}B=-2.4(53.2) \\
\quad p=0.96\end{array}$ & $\begin{array}{c}B=0.008(1.66) \\
\quad p=0.996\end{array}$ \\
\hline RT Var. & $\begin{array}{c}B=-71.7,(14.2) \\
p<0.001 * * *\end{array}$ & $\begin{array}{c}B=-23.0(10.2) \\
p=0.03^{*}\end{array}$ & $\begin{array}{c}B=36.1(12.9) \\
p<0.01^{* *}\end{array}$ & $\begin{array}{c}B=-33.9(12.4), \\
p<0.01^{* *}\end{array}$ & $\begin{array}{c}B=24.1(16.4) \\
p=0.14\end{array}$ & $\begin{array}{c}B=0.13(0.47) \\
p=0.79\end{array}$ \\
\hline Omission errors & $\begin{array}{c}B=-1.40(0.30) \\
p<0.001 * * *\end{array}$ & $\begin{array}{c}B=-0.54(0.20) \\
p=0.01 *\end{array}$ & $\begin{array}{c}B=0.95(0.26) \\
p<0.001 * * *\end{array}$ & $\begin{array}{c}B=-0.18(0.25) \\
p=0.48\end{array}$ & $\begin{array}{l}B=0.38(0.33), \\
\quad p=0.25\end{array}$ & $\begin{array}{c}B=0.004(0.01) \\
p=0.72\end{array}$ \\
\hline Choice errors & $\begin{array}{l}B=-0.18(0.27) \\
p=0.50\end{array}$ & $\begin{array}{l}B=0.11(0.20) \\
\quad p=0.58\end{array}$ & $\begin{array}{c}B=-0.29(0.26) \\
p=0.27\end{array}$ & $\begin{array}{l}B=0.07(0.24) \\
\quad p=0.78\end{array}$ & $\begin{array}{c}B=-0.34(0.31) \\
p=0.28\end{array}$ & $\begin{array}{c}B=-0.001(0.01) \\
p=0.23\end{array}$ \\
\hline
\end{tabular}

SSRT stop-signal reaction time, $R T$ reaction time, Var. variability. B (SE) represents the unstandardized estimate with its standard error, $\gamma_{01}$ the group effect at T2 without reinforcement, $\gamma_{10}$ the time effect in ADHD without reinforcement, $\gamma_{11}$ the interaction effect between group and time without reinforcement, $\gamma_{02}$ the reinforcement effect at T2 in ADHD, $\gamma_{03}$ the interaction effect of group and reinforcement at T2, $\gamma_{04}$ represents the effect of age in boys with $\mathrm{ADHD}$ at $\mathrm{T} 2$ receiving no reinforcement

$* p<0.05, * * p<0.01, * * * p<0.001$

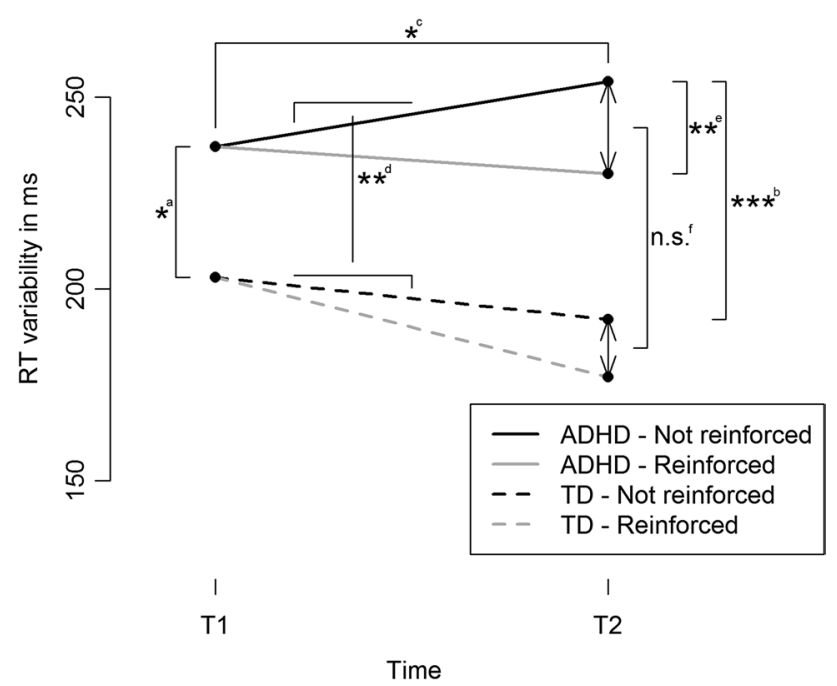

Fig. 1 Reaction time variability as a function of time, group, and reinforcement condition. a effect of group at baseline (T1); $\mathbf{b}$ effect of group at T2 (non-reinforced); $\mathbf{c}$ effect of time in ADHD (non-reinforced); $\mathbf{d}$ time $\times$ group interaction (non-reinforced); $\mathbf{e}$ effect of reinforcement in ADHD; $\mathbf{f}$ group $\times$ reinforcement interaction. ${ }^{*} p<0.05$, $* * p<0.01, * * * p<0.001$. ADHD attention-deficit/hyperactivity disorder, $T D$ typically developing control group, $m s$ milliseconds, n.s. not significant, $R T$ reaction time

variability increased on the second as compared to the first task. The positive $t$ value for the interaction effect indicates that this effect was less pronounced in the TD group. Visual inspection of the effect (Fig. 1) suggests that in the TD group, reaction time variability even decreased in the second as compared to the first task. Similarly, a significant time $\times$ group interaction was observed for omission errors $[t(97.02)=3.70, p<0.001]$. In children with
ADHD, the amount of omission errors increased on the second as compared to the first task (see analyses above), whereas visual inspection suggests that the amount of omission errors in the TD control group even decreased (Fig. 1). No time $\times$ group interactions were found for SSRT, mean reaction time, and choice errors. To sum up, this indicates larger time-on-task effects in the ADHD group than in the TD control group on indices of attention, but not inhibition.

Effects of reinforcement in ADHD (T2: reinforced vs. not reinforced)

Children with ADHD who were vs. were not reinforced were characterized by lower RT variability [ $t$ $(91.95)=-2.73, p=0.008]$. Reinforcement did not influence SSRT, mean reaction time, omission errors, and choice errors. This indicates that children with ADHD performed better when they were reinforced as compared to when they were not reinforced, but only with regard to RT variability.

Effects of reinforcement in ADHD vs. TD controls (T2: reinforced vs. not reinforced)

No significant group $\times$ reinforcement effect was found for all measures, indicating that reinforcement equally influenced children with and without ADHD.

\section{Discussion}

Dual pathway models of ADHD and depletion theories were combined to investigate potential depletion of 
executive resources and depletion of motivation in children with and without ADHD. The SST was administered twice in school-aged children with and without ADHD, in which half of the participants was reinforced during the second task. We hypothesized (1) degraded performance of children with ADHD as compared to typically developing (TD) children on the first task and the second task without reinforcement. Moreover, we expected (2a) degraded performance on the second task without reinforcement, as compared to the first task and (2b), and we expected this effect to be larger in children with ADHD than in TD children. We expected (3a) improved performance on the second task with reinforcement, as compared to the second task without reinforcement and (3b), and we expected that children with ADHD profited more from reinforcement than TD children. Performance was measured at two levels, with response inhibition as core higher order executive function, and at a more basic pre-executive level [13], attentional indices as reaction time (RT), RT variability, and errors.

With respect to the first hypothesis, at baseline, groups differed with regard to reaction time (RT) variability and omission errors: children with ADHD were characterized by larger RT variability and made more omission errors than children without ADHD. On the second task without reinforcement, groups differed on RT variability and omission errors: children with ADHD had larger RT variability and made more omission errors than children without ADHD.

No difference between groups was found for SSRT. Although the typical finding of inhibitory differences between children with and without ADHD is quite robust $[9,16,48,49]$, there are other studies that did not find this difference either [50-53].

Potential explanations might relate to participant characteristics. For example, only a subgroup of children with ADHD is characterized by inhibitory deficits [54-56]. Furthermore, comorbidity profiles seem to play a role. For instance, no inhibitory differences were found between control children and children with only ADHD (i.e., without comorbid disorders; [52]). In our sample, comorbidity occurred less frequently than generally described in ADHD: $57 \%$ of our ADHD group had no comorbid disorder, whereas other literature indicates that approximately two-third of the children with ADHD have at least one other comorbid disorder [4]. In line with this explanation, the average SSRT at baseline in our sample was $254 \mathrm{~ms}$ for controls, $274 \mathrm{~ms}$ for children with ADHD without comorbid disorders, and $293 \mathrm{~ms}$ for children with ADHD comorbid disorder(s). An additional analysis proved that children with ADHD without comorbid disorder(s) did not differ from controls with regard to SSRT at T1 $[F(1,76)=1.4$, $p=0.24]$, whereas children with ADHD and comorbid disorder(s) had higher SSRTs than controls $[F(1,70)=4.7$, $p=0.03]^{7}$

Relatedly, Daugherty and colleagues [51] explain differences between studies in terms of severity of the clinical group, where more severe ADHD groups are most likely to show pronounced inhibitory dysfunctioning. In sum, lower comorbidity rates as well as lower severity of ADHD symptoms (e.g., we included four children with ADHD not otherwise specified) might be an explanation for the absence of inhibition effects between groups.

However, increased RT variability in children with ADHD as compared to TD control children was apparent both at baseline and on the second administration without reinforcement. This concurs with a large body of literature, arguing that increased RT variability is a typical and robust finding in ADHD (see [57] for an excellent extensive meta-analytic review). Some theorists even argue that RT variability is a causal mechanism in the existence of ADHD (e.g., Default Mode Network Model [21]), while others regard this variability as the result of other underlying mechanisms, such as behavioral inhibition [14, 57].

With respect to the second hypothesis, the expected time-on-task effect in ADHD was partially confirmed: RT variability and omission errors increased on the second task as compared to the first in the ADHD group. In line with our hypothesis, this time-on-task effect on RT variability and omission errors was larger in the ADHD than in the TD control group (in fact, the TD control group did not deteriorate at all). RT variability and omission errors have been linked to problems with sustained attention and attentional lapses [39, 40, 42, 58]. All together, these results indicate that time-on-task effects in ADHD mainly seem to occur within the domain of basic attention, and not on response inhibition.

With respect to the third hypothesis, the effect of reinforcement in ADHD was partly as expected: reinforced children with ADHD had a smaller RT variability as compared to children with ADHD who were not reinforced in the second task, which might indicate that reinforcement prevented attentional lapses. This finding is in line with a recent meta-analytic review that found small improvements in RT variability as a result of external reinforcement [57].

However, no effect of reinforcement was found on all other outcome indices. This implies that, among all outcome variables, RT variability might be particularly sensitive to the effects of reinforcement. Furthermore, discordant to expectations, reinforcement effects did not differ between groups for all indices, implying that children with ADHD did not profit more from reinforcement than their typically

\footnotetext{
7 Note however that children with ADHD with and without comorbidity did not differ significantly on SSRT at baseline.
} 
developing peers. This contradicts several studies, reporting that children with ADHD profited more from reinforcement (see [16] for a meta-analysis), but concurs with others that also did not find such differences between ADHD and typically developing controls [53, 59-63]. Moreover, despite the overall significant effect, the same meta-analysis showed that only $24 \%$ of the studies found a larger reward sensitivity in ADHD as compared to controls [16].

One explanation for the limited effects of reinforcement could be that reinforcement in the current study was not strong enough. Although the gift was emotionally appealing, it was only worth 50 eurocents. Children with ADHD are assumed to have an elevated reward threshold [18, 64], and as shown by Dovis and colleagues [22], only relatively large rewards (above threshold) motivated children with ADHD enough to improve their performance on EF tasks, whereas small rewards did not exert any influence on performance. A second explanation for the absence of pronounced motivation effects is that several studies have pointed out that only a minority of children with ADHD shows abnormal sensitivity to reinforcement $[65,66]$, possibly explaining the limited reinforcement effects at group level in the current study.

To summarize, stronger time-on-task effects were found in children with ADHD as compared to children without ADHD on indices of basic attention (i.e., RT variability and omission errors). In terms of depletion theories, reinforcement prevented a time-on-task effect on RT variability, implying that the time-on-task effect in the non-reinforced condition could be, at least partly, explained by a depletion of motivation. On the other hand, reinforcement did not affect the time-on-task effect on omission errors, implying that this time-on-task effect could be driven by a depletion of executive resources. Finally, no time-on-task effects were found on other indices, among which inhibition. This implies that, compared to lower level attentional capacities, higher order executive functions, such as inhibition, seem to be less susceptible to the effects of depletion of executive resources and depletion of motivation.

In the current study, slower mean RTs and higher RT variability (i.e., derived from a Gaussian model with two parameters: mean and variance) were interpreted as potential indicators of attention lapses. However, as attention lapses produce a skew in RT distributions, an ex-Gaussian model might be more appropriate, in which a third parameter $(\tau)$ indexes this skew specifically [67] (note, however, that the $\tau$ parameter could be interpreted as an indicator for many different cognitive processes [68]). Therefore, all multilevel analyses were also performed for $\tau$ as an index for attention lapses. ${ }^{8}$ The only significant finding was related to reinforcement: more

8 These analyses were performed using the retimes package in $\mathrm{R}$. attentional lapses were reported when participants were not reinforced instead of reinforced at T2 [t $(91.24)=-2.02, p<0.05]$. Therefore, this additional analysis partly supports our conclusion that problems (in this case attention lapses) originating in a depletion of motivation can be counteracted with reinforcement.

The current results should be considered in the light of four limitations. First, 15 children were excluded, 11 of them because of aberrant performance. Nine of those 11 were diagnosed with ADHD, and a majority dropped out on the second task (mostly without reinforcement). Conceivably, these excluded participants had most pronounced symptoms, and effects were larger if data from these children were taken into account (see Appendix 3 for results without excluding any of the participants). These testing difficulties demonstrate an obvious limitation of the depletion paradigm, in which multiple monotonous tasks are administered in succession, in samples with ADHD. This is in line with a recent meta-analytic review, which described an increase in core ADHD symptomatology (i.e., hyperactivity) in highly cognitive demanding situations as compared to situations with low cognitive demands [69]. Therefore, on the other hand, the current depletion paradigm might be an promising way of testing, mirroring the daily life routine at school, and creating a situation in which ADHD symptoms come to light easier.

Second, it would have been more optimal to diagnose ADHD participants by means of a semi-structured clinical interview, as the currently used clinical diagnoses might be more lenient and potentially included some children with 'subclinical' ADHD. Relatedly, clinical diagnoses might be subject to biases [70]. However, it should be noted that stricter diagnostics and exclusion of subclinical ADHD participants would logically result in larger and not smaller differences between typically developing controls and ADHD subjects. This limitation could also apply to the control group, in which potential neuropsychiatric problems could have been missed. However, the average SSRT of the control group (at T1, SSRT $=254 \mathrm{~ms}$ ) was consistent with typical control group SSRTs reported in a metaanalysis ([11]; the mean SSRT in control groups on similar SSTs was $284 \mathrm{~ms}(k=24, n=937)$. Hence, inhibitory problems in the control group seem unlikely.

Third, the absence of reinforcement effects is interpreted as evidence in favor of executive over motivational depletion. However, self-evidently, another explanation for the absence of those effects could be that statistical power was not high enough. Therefore, these results should be interpreted with caution.

Fourth, the validity of the SSRT as a pure index of response inhibition is subject to debate. This SSRT deficit might reflect general attentional or cognitive processes that go beyond purely inhibitory processes $[8,49]$. However, on 
a more theoretical level, the current SST is, in our opinion, the best index to assess response inhibition. First, it directly taps into the construct of interest and measures inhibition over a longer time period. Second, a meta-analysis on response inhibition differences between ADHD and controls reviewing 41 studies showed that between group differences (i.e., ADHD groups showing stronger inhibitory deficits) were most pronounced when responses, as in the current study, were spatially noncompatible [11]. Although note that some of the studies reported in that meta-analysis also reported no group differences in SSRT, as in the current study. Third, an additional advantage of the SST is that it derives both measures for inhibition and for attention.

The current study has several implications, for future research as well as for clinical practice. EF was assessed under depleting circumstances, which matches most children's daily school routine, in which they have to use their EFs over prolonged periods of time. This is not to say that we advocate the SST as an ecologically valid EF instrument, as laboratory EF tasks do not correlate well with real life measures [71] and the task that was used in the current study was more repetitive than regular schoolwork.

Our results showed that attentional problems in ADHD became more apparent in the second task. Moreover, the relatively large drop-out in our study potentially indicates that core psychiatric symptoms might come to light easier after a certain amount of time. Hence, we recommend future research to further investigate the diagnostic value of this paradigm in clinical samples.

With regard to treatment of ADHD, psycho-education to children with ADHD and their parents and teachers should emphasize the tendency for problems to increase after prolonged exertion. If children recognize depletion, they can be taught to adjust their behavior accordingly, for example, by taking a short break or switch to less demanding tasks. Furthermore, we suggest therapists to keep their sessions short and offer short breaks, to prevent attentional lapses. If time-on-task effects occur, for example, during school work, this can be partly counteracted with reinforcement.

The current approach was adopted to distinguish between depletion of executive resources and depletion of motivation. However, recent studies show that children with ADHD show heterogeneous patterns of deficits, some having especially deficits in executive functioning, whereas in others, mainly, motivational aberrations are observed [56, $65,66,72,73]$. Consequently, the use of a design comparing ADHD and control groups limits the possibility to elucidate this heterogeneity within the ADHD group. A more personalized approach, in which individual deficits could be assessed and, eventually, treated, would be a promising addition to the literature and is in line with current trends in youth mental healthcare ([73]; see [74] for an extensive review on personalized interventions for youth).
In sum, children with ADHD are more affected timeon-task effects than TD controls, as shown on measures of inattention (RT variability and omission errors), but not inhibition. These time-on-task effects seem to originate in a depletion of executive resources as well as a depletion of motivation. Offering external reinforcement is a promising way to compensate for depletion of motivation and, consequently, to prevent attention lapses in children with ADHD. The depletion paradigm offers both a new perspective on diagnostic assessment of ADHD and provides further clues for optimizing treatment of children with ADHD.

Acknowledgements We would like to thank participating schools, children, and their parents for participation. Furthermore, we would like to express our gratitude to De Bascule, Academic Center for Child and Adolescent Psychiatry for the recruitment of children with ADHD.

\section{Compliance with ethical standards}

Funding This research is supported by a VICI grant (453-12-005) of the last author from the Netherlands Organization for Scientific Research (NWO). JAR is supported by grant MaGW (480-12-015) awarded by the Netherlands organization for scientific research (NWO). The funding source had no role in the study design, collection, analysis or interpretation of the data, writing the manuscript, nor the decision to submit the paper for publication.

Conflict of interest All authors declare that they have no conflict of interest.

Research involving human participants All procedures performed were in accordance with the ethical standards of the institutional research committee and with the 1964 Helsinki declaration and its later amendments or comparable ethical standards.

Informed consent Informed consent was obtained from primary caretakers of all children included in the study.

Open Access This article is distributed under the terms of the Creative Commons Attribution 4.0 International License (http://creativecommons.org/licenses/by/4.0/), which permits unrestricted use, distribution, and reproduction in any medium, provided you give appropriate credit to the original author(s) and the source, provide a link to the Creative Commons license, and indicate if changes were made.

\section{References}

1. Daley D, Birchwood J (2010) ADHD and academic performance: why does ADHD impact on academic performance and what can be done to support ADHD children in the classroom? Child Care Hlth Dev 36:455-464

2. Nigg JT (2013) Attention-deficit/hyperactivity disorder and adverse health outcomes. Clin Psychol Rev 33:215-228

3. Klassen AF, Miller A, Fine S (2004) Health-related quality of life in children and adolescents who have a 
diagnosis of attention-deficit/hyperactivity disorder. Pediatrics 114:e541-e547

4. Jensen PS, Hinshaw SP, Kraemer HC, Lenora N, Newcorn JH, Abikoff $\mathrm{HB}$ et al (2001) ADHD comorbidity findings from the MTA study: comparing comorbid subgroups. J Am Acad Child Psy 40:147-158

5. Sonuga-Barke EJ, Coghill D (2014) Editorial perspective: laying the foundations for next generation models of ADHD neuropsychology. J Child Psychol Psyc 55:1215-1217

6. Coghill DR, Seth S, Matthews K (2014) A comprehensive assessment of memory, delay aversion, timing, inhibition, decision making and variability in attention deficit hyperactivity disorder: advancing beyond the three-pathway models. Psychol Med 44:1989-2001

7. Sonuga-Barke EJ (2003) The dual pathway model of AD/HD: an elaboration of neuro-developmental characteristics. Neurosci Biobehav R 27:593-604

8. Alderson RM, Rapport MD, Kofler MJ (2007) Attention-deficit/ hyperactivity disorder and behavioral inhibition: a meta-analytic review of the stop-signal paradigm. J Abnorm Child Psych $35: 745-758$

9. Willcutt EG, Doyle AE, Nigg JT, Faraone SV, Pennington BF (2005) Validity of the executive function theory of attention-deficit/hyperactivity disorder: a meta-analytic review. Biol Psychiat 57:1336-1346

10. Martinussen R, Hayden J, Hogg-Johnson S, Tannock R (2005) A meta-analysis of working memory impairments in children with attention-deficit/hyperactivity disorder. J Am Acad Child Psy 44:377-384

11. Huizenga HM, van Bers BM, Plat J, van den Wildenberg WP, van der Molen MW (2009) Task complexity enhances response inhibition deficits in childhood and adolescent attention-deficit/ hyperactivity disorder: a meta-regression analysis. Biol Psychiat 65:39-45

12. Miyake A, Friedman NP (2012) The nature and organization of individual differences in executive functions four general conclusions. Cur Dir Psyc Sci 21:8-14

13. Barkley RA (2012) Executive functions What they are, how they work, and why they evolved. Guilford Press, New York

14. Barkley RA (1997) Behavioral inhibition, sustained attention, and executive functions: constructing a unifying theory of ADHD. Psychol Bull 121:65-94

15. Luman M, Tripp G, Scheres A (2010) Identifying the neurobiology of altered reinforcement sensitivity in ADHD: a review and research agenda. Neurosci Biobeh Rev 34:744-754

16. Ma I, van Duijvenvoorde A, Scheres A (2016) The interaction between reinforcement and inhibitory control in ADHD: a review and research guidelines. Clin Psyc Rev 44:94-111

17. Luman M, van Meel CS, Oosterlaan J, Geurts HM (2012) Reward and punishment sensitivity in children with ADHD: validating the sensitivity to punishment and sensitivity to reward questionnaire for children (SPSRQ-C). J Abn Child Psychol 40:145-157

18. Luman M, Oosterlaan J, Sergeant JA (2005) The impact of reinforcement contingencies on AD/HD: a review and theoretical appraisal. Clin Psychol Rev 25:183-213

19. Johnson KA, Kelly SP, Bellgrove MA, Barry E, Cox M, Gill M, Robertson IH (2007) Response variability in attention deficit hyperactivity disorder: evidence for neuropsychological heterogeneity. Neuropsychologia 45:630-638

20. Swaab-Barneveld H, De Sonneville L, Cohen-Kettenis P, Gielen A, Buitelaar J, van Engeland H (2000) Visual sustained attention in a child psychiatric population. J Am Acad Child Psy 39:651-659

21. Sonuga-Barke EJ, Castellanos FX (2007) Spontaneous attentional fluctuations in impaired states and pathological conditions: a neurobiological hypothesis. Neurosci Biobehav R 31:977-986
22. Dovis S, van der Oord S, Wiers RW, Prins PJM (2012) Can motivation normalize working memory and task persistence in children with attention-deficit/hyperactivity disorder? The effects of money and computer-gaming. J Abnorm Child Psych 40:669-681

23. Hofmann W, Schmeichel BJ, Baddeley AD (2012) Executive functions and self-regulation. Trends Cogn Sci 16:174-180

24. Muraven M, Baumeister RF (2000) Self-regulation and depletion of limited resources: does self-control resemble a muscle? Psychol Bull 126:247-259

25. Baumeister RF, Vohs KD, Tice DM (2007) The strength model of self-control. Cur Dir Psychol Sci 16:351-355

26. Hagger MS, Wood C, Stiff C, Chatzisarantis NL (2010) Ego depletion and the strength model of self-control: a meta-analysis. Psychol Bull 136:495-525

27. Inzlicht M, Schmeichel BJ (2012) What is ego depletion? Toward a mechanistic revision of the resource model of selfcontrol. Perspect Psychol Sci 7:450-463

28. Huizenga HM, van der Molen MW, Bexkens A, Bos MG, van den Wildenberg WP (2012) Muscle or motivation? A stop-signal study on the effects of sequential cognitive control. Front Psychol 3:126

29. Muraven M, Slessareva E (2003) Mechanisms of self-control failure: motivation and limited resources. Pers Soc Psychol B 29:894-906

30. Logan GD, Cowan WB (1984) On the ability to inhibit thought and action: a theory of an act of control. Psychol Rev 91:295-327

31. Logan GD (1994) On the ability to inhibit thought and action: a users guide to the stop signal paradigm. In: Dagenbach D, Carr TH (eds) Inhibitory processes in attention, memory, and language. Academic Press, San Diego, pp 189-239

32. American Psychiatric Association (2000) Diagnostic and statistical manual of mental disorders, 4th revised edition (DSMIV-TR). American Psychiatric Association, Washington, DC

33. Greenhill LL (1998) Childhood attention deficit hyperactivity disorder: Pharmacological treatments. In: Nathan PE, Gorman J (eds) A guide to treatments that work. Oxford University Press, New York, pp 42-64

34. Soreni N, Crosbie J, Ickowicz A, Schachar R (2009) Stop signal and conners' continuous performance tasks test-retest reliability of two inhibition measures in ADHD children. J Attention Disord 13:137-143

35. Solanto MV, Abikoff H, Sonuga-Barke E, Schachar R, Logan GD, Wigal T, Turkel E (2001) The ecological validity of delay aversion and response inhibition as measures of impulsivity in AD/HD: a supplement to the NIMH multimodal treatment study of AD/HD. J Abnorm Child Psych 29:215-228

36. Tillman CM, Thorell LB, Brocki KC, Bohlin G (2007) Motor response inhibition and execution in the stop-signal task: development and relation to ADHD behaviors. Child Neuropsychol 14:42-59

37. Pliszka SR, Borcherding SH, Spratley K, Leon S, Irick S (1997) Measuring inhibitory control in children. J Dev Behav Pediat 18:254-259

38. Nigg JT (1999) The ADHD response-inhibition deficit as measured by the stop task: replication with DSM-IV combined type, extension, and qualification. J Abnorm Child Psych 27:393-402

39. Winstanley CA, Eagle DM, Robbins TW (2006) Behavioral models of impulsivity in relation to ADHD: translation between clinical and preclinical studies. Clin Psychol Rev 26:379-395

40. Bezdjian S, Baker LA, Lozano DI, Raine A (2009) Assessing inattention and impulsivity in children during the Go/NoGo task. Brit J Dev Psychol 27:365-383

41. Leth-Steensen C, Elbaz ZK, Douglas VI (2000) Mean response times, variability, and skew in the responding of ADHD 
children: a response time distributional approach. Acta Psychol 104:167-190

42. Vaurio RG, Simmonds DJ, Mostofsky SH (2009) Increased intra-individual reaction time variability in attention-deficit/ hyperactivity disorder across response inhibition tasks with different cognitive demands. Neuropsychol 47:2389-2396

43. Doran SM, van Dongen HPA, Dinges DF (2001) Sustained attention performance during sleep deprivation: evidence of state instability. Arch Ital Biol 139:253-267

44. Snijders TAB, Bosker RJ (1999) Multilevel analysis: an introduction to basic and advanced multilevel modeling. Sage, Thousand Oaks, CA

45. Zelazo PD, Craik FI, Booth L (2004) Executive function across the life span. Acta Psychol 115:167-183

46. Frazier TW, Demaree HA, Youngstrom EA (2004) Meta-analysis of intellectual and neuropsychological test performance in attention-deficit/hyperactivity disorder. Neuropsychol 18:543-555

47. Dennis M, Francis DJ, Cirino PT, Schachar R, Barnes MA, Fletcher JM (2009) Why IQ is not a covariate in cognitive studies of neurodevelopmental disorders. J Int Neuropsych Soc 15:331-343

48. Oosterlaan J, Logan GD, Sergeant JA (1998) Response inhibition in $\mathrm{AD} / \mathrm{HD}, \mathrm{CD}$, comorbid $\mathrm{AD} / \mathrm{HD}+\mathrm{CD}$, anxious, and control children: a meta-analysis of studies with the stop task. J Child Psychol Psyc 39:411-425

49. Lijffijt M, Kenemans JL, Verbaten MN, van Engeland H (2005) A meta-analytic review of stopping performance in attention-deficit/hyperactivity disorder: deficient inhibitory motor control? J Abnorm Psychol 114:216-222

50. Demurie E, Roeyers H, Wiersema JR, Sonuga-Barke E (2013) No evidence for inhibitory deficits or altered reward processing in ADHD: data from a new integrated monetary incentive delay go/ no-go task. J Attention Disord. doi:10.1177/1087054712473179

51. Daugherty TK, Quay HC, Ramos L (1993) Response perseveration, inhibitory control, and central dopaminergic activity in childhood behavior disorders. J Genet Psychol 154:177-188

52. Jennings JR, van der Molen MW, Pelham W, Debski KB, Hoza B (1997) Inhibition in boys with attention deficit hyperactivity disorder as indexed by heart rate change. Dev Psychol 33:308-318

53. Scheres A, Oosterlaan J, Sergeant JA (2001) Response execution and inhibition in children with $\mathrm{AD} / \mathrm{HD}$ and other disruptive disorders: the role of behavioural activation. J Child Psychol Psyc 42:347-357

54. Sonuga-Barke EJ (2002) Psychological heterogeneity in AD/ HD - a dual pathway model of behaviour and cognition. Beh Brain Res 130:29-36

55. Crosbie J, Schachar R (2014) Deficient inhibition as a marker for familial ADHD. Am J Psychiat 158:1884-1890

56. Nigg JT, Willcutt EG, Doyle AE, Sonuga-Barke EJ (2005) Causal heterogeneity in attention-deficit/hyperactivity disorder: do we need neuropsychologically impaired subtypes? Biol Psychiat 57:1224-1230

57. Kofler MJ, Rapport MD, Sarver DE, Raiker JS, Orban SA, Friedman LM, Kolomeyer EG (2013) Reaction time variability in ADHD: a meta-analytic review of 319 studies. Clin Psychol Rev 33:795-811

58. O'Connell RG, Bellgrove MA, Dockree PM, Robertson IH (2004) Reduced electrodermal response to errors predicts poor sustained attention performance in attention deficit hyperactivity disorder. NeuroReport 15:2535-2538

59. Oosterlaan J, Sergeant JA (1998) Effects of reward and response cost on response inhibition in $\mathrm{AD} / \mathrm{HD}$, disruptive, anxious, and normal children. J Abnorm Child Psych 26:161-174

60. Shanahan MA, Pennington BF, Willcutt EW (2008) Do motivational incentives reduce the inhibition deficit in ADHD? Dev Neuropsychol 33:137-159

61. Desman C, Petermann F, Hampel P (2008) Deficit in response inhibition in children with attention deficit/hyperactivity disorder (ADHD): impact of motivation? Child Neuropsychol 14:483-503

62. Wodka EL, Mark Mahone E, Blankner JG, Gidley Larson JC, Fotedar S, Denckla MB, Mostofsky SH (2007) Evidence that response inhibition is a primary deficit in ADHD. J Clin ExpNeuropsyc 29:345-356

63. Stevens J, Quittner AL, Zuckerman JB, Moore S (2002) Behavioral inhibition, self-regulation of motivation, and working memory in children with attention deficit hyperactivity disorder. Dev Neuropsychol 21:117-139

64. Haenlein M, Caul WF (1987) Attention deficit disorder with hyperactivity: a specific hypothesis of reward dysfunction. J Am Acad Child Psy 26:356-362

65. Sonuga-Barke E, Bitsakou P, Thompson M (2010) Beyond the dual pathway model: evidence for the dissociation of timing, inhibitory, and delay-related impairments in attention-deficit/ hyperactivity disorder. J Am Acad Child Psy 49:345-355

66. Dovis S, Van der Oord S, Huizenga HM, Wiers RW, Prins PJM (2015) Prevalence and diagnostic validity of motivational impairments and deficits in visuospatial short-term memory and working memory in ADHD subtypes. Eur Child and Adoles Psy 24:575-590

67. Lacouture Y, Cousineau D (2008) How to use MATLAB to fit the ex-Gaussian and other probability functions to a distribution of response times. Tutorials Quant Meth Psychol 4:35-45

68. Matzke D, Wagenmakers EJ (2009) Psychological interpretation of the ex-Gaussian and shifted Wald parameters: a diffusion model analysis. Psychon B Rev 16:798-817

69. Kofler MJ, Raiker JS, Sarver DE, Well EL, Soto EF (2016) Is hyperactivity ubiquitous in ADHD or dependent on environmental demands? Evidence from meta-analysis. Clin Psychol Rev 46:12-24

70. Garb HN (2006) The conjunction effect and clinical judgment. J Soc Clin Psychol 25:1048-1056

71. Toplak ME, West RF, Stanovich KE (2013) Practitioner review: do performance-based measures and ratings of executive function assess the same construct? J Child Psychol Psyc 54:131-143

72. Fair DA, Bathula D, Nikolas MA, Nigg JT (2012) Distinct neuropsychological subgroups in typically developing youth inform heterogeneity in children with ADHD. PNAS 109:6769-6774

73. De Zeeuw P, Weusten J, van Dijk S, van Belle J, Durston S (2012) Deficits in cognitive control, timing and reward sensitivity appear to be dissociable in ADHD. PLoS ONE 7:e51416

74. Ng MY, Weisz JR (2016) Annual research review: building a science of personalised intervention for youth mental health. $\mathrm{J}$ Child Psychol Psyc 57:216-236 